\title{
A Metaphor That Keeps on Ticking: The 'Clock' as a Driving Force in the History of Chronobiology Research
}

\author{
Rona Aviram*† and Gal Manella*‡
}

\begin{abstract}
Scientific research is more than just data collection; rather, it inherently relies on interpretation of observations and communicating ideas-all performed through language. Therefore, the terminology used in a certain field is of high importance for analysis of its workings and growth. Here, we applied methods from the metaphor-research field to analyze the history of chronobiology, revisiting a vast corpus of circadian scientific literature published from the 19th century until today. Several metaphors, predominantly that of the 'clock', were used throughout this period. We demonstrate the importance of these metaphors in facilitating scientific understanding, experimental design, and the construction of the field's main paradigms. Taken together, our findings provide what we believe is a much-needed reflection on the role of language in scientific practice. Renewing awareness of these metaphors can help reveal the limitations of the current scientific language and stimulate the search for new terminology and perspectives.
\end{abstract}

\section{Keywords}

circadian rhythm $\bullet$ circadian clock $\bullet$ conceptual metaphor $\bullet$ history and philosophy of science $\bullet$ metaphors in science $\bullet$ teleonomy

\section{Prologue}

In classical and medieval times much of the physical world was considered to have will and agency, similar to living entities. However, this view was overturned with the successful emergence of exact sciences in the 17 th century, in what is now termed the Scientific Revolution. This standpoint viewed the universe as inherently inanimate and mindless, and everything in it was held to be devoid of all agency. Accordingly, the physical world could and should be explainable through deterministic and mechanistic terminology (Westfall, Basalla, and Hannaway 1977).

*The Department of Biomolecular Sciences, Weizmann Institute of Science, Rehovot, Israel

$\dagger$ anorona@gmail.com (iD https://orcid.org/0000-0001-5544-3552

‡ gal.manella@weizmann.ac.il] (i) https://orcid.org/0000-0002-2642-3568

Received 16 January 2020; Revised 1 June 2020; Accepted 4 June 2020 doi:10.3998/ptpbio.16039257.0012.007

๑ OPEN ACCESS - PTPBIO.ORG 
Clocks were the state-of-the-art technology of that time, and they became perhaps the most iconic metaphor of the mechanistic worldview. It served as the leading representation for complex systems, those capable of deterministic, predictable, and self-sustained motion. Newtonian mechanics, by successfully explaining motion of this type, lent its support to the perception of the whole universe as an enormous clock, created by the divine watchmaker. This analogy, called "the clockwork universe", resonated amongst many of the time's leading scientists, including Isaac Newton, Robert Boyle and others (Dolnick 2014; Haber 1975).

In line with this revolution, mechanical analogies such as clocks were gradually applied also to living things and biological functions. For example, in his "Treatise on Man", René Descartes wrote: "These functions (including passion, memory, and imagination) follow from the mere arrangement of the machine's organs every bit as naturally as the movements of a clock or other automaton follow from the arrangement of its counter-weights and wheels" (Carriero et al. 1990).

From the mid-17th century, the 'clock' was employed as a metaphor in various ways. As young scientists in the chronobiology field, we couldn't overlook the extensive use of the 'clock' metaphor in describing biological, daily, rhythmic processes (i.e., circadian rhythms). We wondered: How did this metaphor emerge? Which additional metaphors were in use in chronobiology history, and how were all of these utilized by scientists?

Relying on the foundations of metaphor research and philosophy of science, we revisited circadian scientific literature and historical testimonies of chronobiologists to address the manner in which these metaphors changed throughout the field's history, and how this in turn has influenced research and scientific activity.

\title{
2 The Ticking Metaphor
}

\begin{abstract}
Not only plants, but also animals and humans, have a biological clock that helps to prepare our physiology for the fuctuations of the day. This regular adaptation is referred to as the circadian rhythm, originating from the Latin words circa meaning 'around' and dies meaning 'day'.
\end{abstract}

-The 2017 Physiology or Medicine Nobel Prize announcement

Though a common theme in Western society and an irreplaceable part of today's terminology, the use of 'clock' in relation to circadian phenomena was rare and anecdotal from the dawn of chronobiology in the 18th century and up until the 1950s.

An early use of the 'clock' in regards to biological circadian phenomena was made by JulienJoseph Virey who described "a sort of living clock, put together by nature, and entrained by the fast movement of the sun" (Virey 1814, translated from French in Reinberg, Lewy, and Smolensky 2001). Over a century later, Maynard Johnson (1939) described mouse behavior as "an exceptionally substantial and durable self winding and self regulating physiological clock".

The 'clock' metaphor was generally adopted by the scientific chronobiology community only during the 1950s, in an active, intentional process led by Colin Pittendrigh. Recognized as one of the founding fathers of modern chronobiology, Pittendrigh was an evolutionary biologist who was said to be constantly inspired by the adaptive consequences of biological phenomena (Daan 2010; J. Woodland Hastings 2001). Notably, Pittendrigh (1993) emphasized the significance of purposeful argumentation in biological studies. He coined the term 'teleonomy' to describe a purposeful phenomenon that does not require the presupposition of agency (in contrast to teleology, which associates with agency), for example, for all those traits which evolved in Darwinian fashion. The long-standing controversy of teleology in biology aside (Mayr 1961),

๑ OPEN ACCESS - PTPBIO.ORG 
it is important to highlight this aspect in Pittendrigh's approach as one examines the emergence of the 'clock' metaphor.

In his writings, Pittendrigh reflects on one such purpose-inspired idea, after attending a lecture by Gustav Kramer in 1952 (Pittendrigh 1993). Kramer described new findings on starlings' navigation, which suggested that the birds continuously predict the movement of the sun, 15 angles counter clockwise every hour, thus allowing them to compensate for the angle to correctly estimate directionality. This would require the birds to have the ability to measure the passage of time. In the same year, Kramer (1952) described his view in a paper using the words "the following 'devices' in the bird's central nervous system were postulated at an early stage: an intrinsic timepiece and also a correct estimation of the azimuth movement of the sun" (emphasis added).

Following Kramer's lecture, Pittendrigh described experiencing a eureka moment: He realized that this capability, and purpose, of time-measurement can potentially explain a wide range of circadian observations, both mechanistically and evolutionarily. To express the full force of this generalization, Pittendrigh deliberately used the term 'clock'. In his 1954 paper, Pittendrigh already invokes the term to describe a widespread internal biological mechanism. For example, its opening sentence declares: "Clocks, processes measuring absolute time, occur in living organisms” (Pittendrigh 1954).

From then on, the 'clock' metaphor propagated rapidly throughout the field's discourse and literature of the 1950s. By the seminal Cold Spring Harbor Symposium of 1960, entitled "Biological Clocks", the metaphor had become ubiquitous and was extensively used in most of the lectures (Cold Spring Harbor Laboratory of Quantitative Biology 1960). Researchers who had hitherto studied seemingly unrelated subjects, from bird navigation to plant-leaf movements, were now gathered together by the recognition that they were looking at the very same thingthe circadian clock (Schwartz and Daan 2017). This event symbolizes how the term 'clock' served as a foundational and unifying concept of the field of chronobiology.

\section{The Conceptual Metaphor}

\section{Time flies like an arrow; fruit fies like a banana.}

\section{-Groucho Marx/Anonymous}

To fully understand the influence of the 'clock' metaphor on scientific research, it is necessary to discuss the unique position metaphors hold in our language and life. The linguistics literature covers a wide range of metaphorical uses in human language. This chapter is not intended to comprehensively describe them all, but rather highlight those which served as the basis for our study.

Classically, metaphors are considered as a type of figurative language, wherein a literal statement is substituted with a more "aesthetically appealing" symbolic term, and more specifically as an implicit comparison between two subjects. In contrast, from the middle of the 20th century, Max Black $(1962,1979)$ and others revised this view of metaphors, emphasizing a dynamic interaction between systems of implications associated with the two subjects. This process is (metaphorically) described as mapping, or projecting, of some characteristics from a "source domain" to a "target domain".

In their seminal study, George Lakoff and Mark Johnson demonstrated the fundamental role metaphors serve in human language, thought, and action (Lakoff and Johnson 1980). They show that in order to speak about abstract concepts, one must necessarily use metaphors from more familiar domains. These metaphors are called "conceptual metaphors". For instance, 'time' is

๑ OPEN ACCESS - PTPBIO.ORG 
a ubiquitous - albeit abstract - concept. In order to speak about time, we usually use metaphors of a moving object/fluid, or of money/resource. Examples are: "time flies", "the future is $a p$ proaching", "don't waste my time", "time is running out". These expressions are often taken literally in everyday language; however, they stem from a metaphorical concept of time, as time is neither money, nor a fluid.

Since saying 'time is money' does not explain what about time is like money, an analytical tool is required in order to dig deeper into the interactions between a metaphor and its subject. This can be achieved by analyzing the metaphors' entailments. Originally described by Lakoff and Johnson, entailments are attributes and associations of the metaphorical term which can be assigned to the metaphor's subject. For example, the metaphor time is money entails that:

1. Time has quantity.

2. Time is valuable.

3. Time can be used/spent/invested.

4. Time can be lost/saved.

The entailments rely on seemingly logical argumentation, e.g., (i) money is valuable, and (ii) time is money, therefore (iii) time is valuable.

In this way, the metaphor of money grants meaning to the concept of time. Therefore, by exposing the metaphor's entailments, one could detect which features of the metaphor are used, and what is lost or gained in a specific context. Moreover, entailments can be used to compare different metaphors. For example, time is a moving object entails a very different set of entailments, e.g.:

1. Time has a position relative to us.

2. Time has directionality.

3. Time has speed.

Evidently, neither of these two metaphors fully capture the essence of time, and using either one will impose a very different, if not contradictory, view of it. Together, these examples show how the analysis of the metaphors' entailments can be used to reveal the manner in which different conceptual metaphors grant different meanings to their subject.

The 'clock', as used in the chronobiology field, is clearly a conceptual metaphor: literally there is no clock; rather there are some characteristics, or entailments, pertaining to man-made timepieces that can be mapped onto the observation domain. As the subjects of scientific research are often abstract, metaphors in scientific language have been the focus of several scholarly works throughout the years (Hesse 1970; Boyd 1979; Brown 2003). Therefore, we propose to use the conceptual metaphor theory as a theoretical framework for studying how the 'clock' metaphor was used in scientific processes. In the next sections, we integrate entailment-drawing as our main methodology to analyze the 'clock' metaphor's roles in chronobiology research, and we dissect the evolution of the metaphorical language of the field.

\section{The Useful Metaphor}

There is no science without fancy, and no art without facts.

-Vladimir Nabokov (1973)

Relying on the foundations of the conceptual metaphor theory, we analyzed texts from the 19th century to the present, including scientific publications, transcripts of conferences, and written testimonies of chronobiologists. We found that the 'clock' metaphor has had two important 
consequences in research: First, the metaphor introduced new meaning to its subject, thereby enabling the explanation of an elusive phenomenon in terms of a well-known one (an explanatory mean). Second, metaphor's entailments can be combined into hypotheses and experimental designs (a predictive mean).

These utilities may be uncovered by analyzing the 'clock' metaphor's entailments, and seeing how they played out through the field's history. In this section, we will examine the entailments which appeared early in the field's history in tandem with its establishment.

\section{(1) A clock is automatic/self-sustained.}

From the early experiments of Jean Jacques De Mairan (1729), circadian rhythms were shown to persist in constant darkness, indicating they are self-sustained. This became a fundamental and much disputed question in the field: Are those rhythms indeed autonomous, or is there an unknown environmental rhythm which serves as an exogenic driver? The works of Erwin Bunning, Hans Kalmus, and others in the early 20th century tilted the scale towards the autonomous alternative (Bunning 1960; Hans Kalmus 1988; Daan 2010). The acceptance of the 'clock' metaphor supported this exact hypothesis-as a clock is a self-sustained rhythm generator, it therefore facilitated an explanation of this type.

(2) A clock has a purpose: to measure and display time (of day).

As discussed earlier, understanding "what is the purpose" of certain phenomena is an essential part of biological discourse, and often - as was notable in the case of Pittendrigh-serves as a driving force in research.

By using the term 'clock', one adopts a certain perspective on the purpose of the biological system: that it's there in order to measure daily time. Accepting the purpose of time measurement enables explanation of all known circadian rhythms in a meaningful manner (Pittendrigh 1993). In turn, additional entailments arise from this, as they allow the clock to fulfill its purpose:

\section{(2.1) A clock can and should be reset.}

Clocks are useful only when set to the correct time. Therefore, an important question becomes: How is the biological clock reset ("entrained") by the environment? Over the years, chronobiologists aspired to provide answers to this question, and successfully identified timing cues ("zeitgebers") such as light and temperature cycles (Aschoff 1960).

Moreover, the fact that clocks are resettable provided the early chronobiologists with an entry point for dissecting the clock mechanism, albeit indirectly, at a time when it was still treated as a "black box". By changing environmental inputs and observing the subsequent effect on rhythmicity, researchers could gather information which served as the primary empirical source for modeling of the biological oscillator itself (Daan 2010; Winfree 2001; C. H. Johnson 1992).

\section{(2.2) A clock should be reliable.}

Clocks are useful only if their frequency is resistant to environmental changes. Historically, the great leap in the accuracy of marine chronometers was made by designing a temperaturecompensation mechanism (Sobel 2007). In circadian research, the view of the role of temperature compensation has changed throughout the years: initially, in the 1930s and 1940s, experimental evidence suggested the rhythms were temperature-dependent (Hans Kalmus 1940). This outlook complemented what is generally known about the rate of biochemical or metabolic reactions, which change as a function of temperature. Therefore, temperature dependence 
supported the theory of an autonomous, biochemically driven rhythm, as opposed to the exogenous origin hypothesis. However, Pittendrigh (1954), equipped with the 'clock' metaphor, revised this conception and showed through experimentation that the rhythms are actually temperature independent/compensated.

This is the most straightforward example of a predictive entailment. For example, Woody Hastings and Beatrice Sweeney (1957) noted that: "Temperature independence constitutes support for the idea that a diurnal rhythm could be related to an accurate biological timing device, or clock. If, in fact, the rhythms are related to a chronometer mechanism, the significance of temperature independence is clear". In addition, Pittendrigh (1960) in his Cold Spring lecture explained: "This explicit reformulation of rhythms as clocks was mainly responsible for establishing, in the last ten years, the temperature-compensation of their period as a real generalization: it was deliberately sought as a functional prerequisite of a good clock".

Here, we use the term "predictive" in a manner unlike that used with respect to hypotheses and models. In the latter case, predictions are drawn directly and logically, and testing their validity will confirm or reject the model/hypothesis they were drawn from. Generally, the metaphor is not a hypothesis per se, and isn't meant to be directly tested. This is reflected by the fact that a contradiction between a metaphor and the empirical evidence does not necessitate rejection of the metaphor from scientific language. In contrast, a metaphor can be predictive in the sense that it inspires the process of hypothesis making, and consequently the drawing of predictions (see Hesse 1970). As shown here for the 'clock', the metaphor's entailments are the elements to be tested empirically, and not the metaphor itself.

In sum, these are all examples for entailments which are analytically useful, in the sense that they enable new hypotheses to be drawn and new experiments to be designed. There's an argument to be made that the 'clock' metaphor has been accepted and became so widespread exactly because it allowed experimentation and, therefore, scientific progress.

\section{The Restless Metaphor}

\section{Like a rolling stone}

$$
\text { -Bob Dylan (1972) }
$$

The entailments described in the previous section became the basis for the functional definition of a biological clock as it was perceived in the 1950's (Pittendrigh 1960):

\section{The clock is a self-sustained, resettable and temperature-compensated, biological oscillator.}

This initial set of entailments served as what we term "defining entailments", in the sense that they defined what a clock $i s$, what can be regarded as "clock-controlled", and hence what can be studied within the framework of the circadian field. An empirical observation that does not fit in with these entailments would not instigate the whole-cloth rejection of the 'clock' as a concept, but would rather itself be classified as a non-circadian phenomenon by definition.

And yet, language, like science, undergoes constant changes and evolution. And so, later in the growth of the circadian field, additional 'clock' entailments were utilized. For example:

(3) A clock is an object/apparatus, and therefore:

(3.1) A clock has a distinct location;

(3.2) A clock has parts;

(3.3) A clock has confined limits.

๑ OPEN ACCESS - PTPBIO.ORG 
According to these later entailments the clock is a physical machine, and is therefore a system which can be understood through a reductionist approach. This view was manifested throughout the search and subsequent discovery of the location of the clock in a number of model organisms. For example, circadian rhythmicity in rodents was abolished following the lesion of a specific region of the brain, the Suprachiasmatic Nucleus (SCN), (Moore and Eichler 1972; Stephan and Zucker 1972), which became known as the mammalian "master clock". Later on, this location entailment was still useful as scientists discovered that circadian clocks tick in every cell throughout the body (Yoo et al. 2004; Nagoshi et al. 2004; Balsalobre, Damiola, and Schibler 1998).

An even more fundamental implementation of this entailment is reflected in the search for the molecular basis underlying the clock phenomenon (namely, "a clock has parts"). The first of what are now known as "clock genes", the period gene in Drosophila, was described in the seminal paper of Seymour Benzer and Ron Konopka in 1971, and its mutated forms presented disrupted behavioral periodicity (Konopka and Benzer 1971). Later on, the gene was sequenced (P. Reddy et al. 1984), and the model of the negative feedback loop was introduced in order to explain how these genes generate self-sustained oscillations (Hardin, Hall, and Rosbash 1990).

The mere concept of "clock genes" and "clock molecules" (Rosbash and Hall 1989) goes hand in hand with the mechanistic view of the biological clock as an apparatus, which is made of specific genes and their gene products, similar to clockwork. The 2017 Nobel Prize awarded to Jeffrey Hall, Michael Rosbash, and Michael Young, who first established the feedback model in flies, demonstrates the profound significance of this perspective.

Intriguingly, this entailment necessitates a complementary conclusion: if some genes are clock genes then the rest of the genes (or any cellular element for that matter) are not part of the clock - that is, not part of an object that has well defined limits. This seemingly trivial dichotomy requires each newly discovered factor to be classified either as a part of the clock or as something else (such as input component, clock-modulator, accessory loop and so on). Hence, defining the limits of the clock still constitutes a major field of study and debate to this day (Pett et al. 2016; Takahashi 2004; Anafi et al. 2014).

In retrospect, the emergence of the entailments described in this section had a profound impact on the definition of clocks. A more modern definition has become, at least implicitly, structural, and can be phrased as follows:

\section{The clock is the set of genes and molecules, and the relations between them, which control} daily rhythms.

When contemporary chronobiologists refer to "the clock" of a specific organism, they will often mean to refer specifically to the molecular network that was discovered in this particular organism (e.g., Clock, Bmal1, Cry's and Per's are the mammalian circadian clock). This shift in meaning is further manifested in scientific practice. An important example is the manner in which biologists determine empirically if a phenomenon is controlled by the clock-a routine question arising in chrono-research. According to the original, functional definition, a clock-controlled observation is expected to exhibit self-sustainability, resetability, and temperature compensation. In contrast, the structural definition deems a phenomenon clock-controlled if it is abolished or modified in "clock-deficient" mutants. In fact, the later definition permits that even a nonrhythmic phenomenon (such as a health condition) will be defined as clock-controlled as long as it is affected by "clock disruption". Overall, the 'clock is an apparatus' sub-entailments might be viewed as alternative "defining entailments", in that they reshape the limits of the circadian field. 
In conclusion, different entailments can be utilized by different scientists at different times. Some entailments gain a higher level of importance in specific contexts and, as such, allocate different meanings to observation and to the metaphorical term itself. As we will show in the next section, such dynamics are not restricted to a single, specific metaphor, and can occur between different metaphors altogether.

\section{The Retired Metaphor}

$$
\begin{aligned}
& \text { Metaphors make sense of our experience ... [by] highlighting some things and hiding } \\
& \text { others. }
\end{aligned}
$$

-Lakoff and Johnson (1980)

A complete overlap between the metaphor and its target domain can never occur, as that would make the two synonymous. While the use of a metaphor emphasizes similarities between these two domains, and promotes a coherent view and understanding of them, it also necessarily masks the differences between them.

The choice of one specific metaphor favors a certain set of entailments over another. As will be demonstrated below, different metaphors can be applied to similar or identical sets of observations, reflecting different perspectives and priorities of the researchers.

\section{A. The gramophone}

In 1940 Hans Kalmus explicitly used a metaphor of a 'gramophone', when describing rhythms of eclosion in Drosophila, as "a system of recording and reproduction analogous to the gramophone with the following peculiar features: one single record only is manufactured, this process lasting 24 hours. And this record is then repeated day by day, simultaneously recording and reproducing" (Hans Kalmus 1940).

The Kalmus gramophone can both read and write, and is essentially documenting "warnings" from the environment (conceptually similar to the later term "zeitgebers"). Under normal conditions, an organism behaviorally responds to these warnings. Yet, if under certain circumstances a warning is missing from the environment, the gramophone would 'sound' the previously recorded one, and the correct response will occur. Ultimately, this was his suggested mechanism for the persistence of rhythms under constant conditions.

Interestingly, in line with his own results, Kalmus suggested that the speed of the record can change with temperature, so the system is temperature dependent.

Later in his career, Kalmus dismissed his own metaphor, but stipulated its advantage in reflecting an adaptive ("instructive") view of the system (H. Kalmus and Wigglesworth 1960). Indeed, environmental cues can play a much greater role in dictating behavior through the gramophone described above, in contrast with oscillator-metaphors (such as a pendulum or a clock).

\section{B. The tape recorder}

A similar metaphor was echoed in 1975 by James Enright, who was also interested in the dynamics and flexibility of circadian patterns of activity. Following his observations of various rhythmic patterns of behavior of birds and mice, he suggested "the analogy of a 24-hr closed-loop tape recorder" (Enright 1975). Overall, Enright suggested that the purpose of the organism's tape is to repeat and reinforce "a successful day" behavioral pattern. According to this theme, the pattern is modifiable based on experience, as "information" can be recorded or erased. 
Regarding the relations between innate and adaptive aspects of the system, Enright (1975) wrote: "The basic circadian oscillation system is inborn: genetically fixed in period, and not learned. In other words, the length of the circadian tape loop is not determined by experience; hence, if we wish to make analogies with more usual learning processes, we must confine our attention to the messages on the inborn tape loop, which can be recorded or erased, as dictated by experience".

\section{Zeitgedächtnis}

For many decades of the 20th century, researchers from the Karl von Frisch lab, who studied bee activity and feeding behavior, used another alternative term: zeitgedächtnis ("time-memory") (for example see Beling 1929; Renner 1960). According to their terminology, the bees could sense and remember the time-of-day of a visit at a location of a food source, and return to it at the same time during the following days.

Led by this terminology, they chose to follow up on these intriguing findings by examining the environmental factors that might convey such timing information to the bees. In a paper from 1993, Pittendrigh relates to this very issue of language and interpretation: "The use of 'memory' and 'training' seriously distracts attention from the innate components in the overall behavior. And unlike 'clock', the word 'memory' fails to raise the issue of phase or local time" (Pittendrigh 1993).

These alternative metaphors were suggested and utilized in parallel to that of the clock, revealing the personal inclination and worldview of the different researchers who were its proponents. In hindsight, these three metaphors are similar in that they reflect a more adaptive and flexible view of the system, compared to a 'clock' which is innate, autonomous, and rigid. Additionally, they all focus on what we now call "output processes", and almost ignore the "core pacemaker" that generates the underlying frequency.

In contrast, alternative metaphors from the 1990s onward deal with a different aspect altogether:

\section{Temporal program}

In his last years, Pittendrigh, the very person who initially promoted the 'clock', reviewed the empirical evidence gathered up to the 1990s and declared: "The time has come to stop talking about the biological clock [...] that paradigm was useful in its day" (Pittendrigh 1996). Pittendrigh takes issue with the 'clock paradigm' based on conflicts/contradictions he observed, which we will next attempt to reformulate by the entailments methodology:

First, 'clock'-entailment no. 2 (A clock has a purpose: to measure and display time) supposedly distracts researchers from any other, more important, purpose of the system.

Second, even if we accept that this is indeed the purpose, it further entails that:

\section{(2.3) Only one clock is needed.}

This is because in order for telling time there is no advantage in multiplicity of clocks, and it can even create problems (for example, conflicting messages in case of misalignment). However, unequivocal evidence for the multiplicity of oscillators has accumulated throughout the years (Aschoff and Wever 1976; Takahashi and Menaker 1982; Morse, Hastings, and Roenneberg 1994) and proved this entailment to be irrelevant.

In retrospect, the roots of Pittendrigh's "strongly held convictions" can already be seen in his 1960 lecture in Cold Spring Harbor, wherein he declared: "We are forced, in fact, to abandon the common current view that our problem is to isolate and analyze ...'the internal clock,' and 
are faced with the conclusion that the organism comprises a population of quasi-autonomous oscillatory systems" (Pittendrigh 1960).

Therefore, Pittendrigh suggested an alternative term, the 'temporal program'. Based solely on his chronicles, it is challenging to dissect the full meaning of his 'temporal program', and its complete analysis goes well beyond the scope of this paper. In short, it appears that Pittendrigh regards the circadian system as a multi-oscillator system-one whose main purpose is to establish an internal temporal organization, rather than aligning its activity with the external world (which is the purpose of 'clock').

\section{The Upcoming Metaphor}

Music is sound organized in time.

-Edgard Varèse

As of yet, the above alternative metaphors have neither replaced the 'clock', nor been widely adopted in the field's discourse. However, reviewing the literature from the past 20 years reveals many cases in which a new metaphor was in fact added to that of the 'clock': a conductor of an orchestra. Interestingly, the word 'clock' is still very much in use, but it is now supported by this new metaphor, as in sentences like "the clock orchestrates $X$ " or " $Y$ is conducted by the clock".

Clearly, the 'conductor' metaphor is fundamentally different from 'clock', as the former is anthropomorphic and socially driven, while the latter is uber-mechanistic. Taken out of context, the use of both metaphors in the same sentence is somewhat poetic and even surrealistic.

In the field's literature and parlance, we observed the 'conductor' has been mainly used to describe: (1) the SCN as a conductor of peripheral clocks (e.g., Buhr and Takahashi 2013; Davidson, Yamazaki, and Menaker 2003; Dibner, Schibler, and Albrecht 2010), and (2) cellular clocks as orchestrators of cellular processes (e.g., Thurley et al. 2017; Y. Liu et al. 1995; A. B. Reddy et al. 2006; Mauvoisin et al. 2017).

To understand the potential benefits of this new metaphor, we will now examine some of its specific entailments:

(B1) A conductor has a purpose: to coordinate the activity of the orchestra.

The conductor sets the relations between the different players. This aspect can shift the focus from time telling, or absolute time measurement (as entailed by the 'clock' metaphor), to the importance of synchronicity, harmony, coordination, and coherence of processes in time. On some level, this could be similar to the proposed purpose of Pittendrigh's 'temporal program', highlighting the utility of the internal organization (Takahashi, Turek, and Moore 2001).

(B2) A conductor is a part of an orchestra.

Seemingly, the conductor is as singular as the clock, since it doesn't make sense to have more than one conductor. That said, this metaphor allows for a new layer of complexity, which is that of the "players": active, individual, and otherwise autonomous, they hence can be regarded as multiple non-redundant oscillators. According to this view, the peripheral clocks are the 'players', conducted by the central clock, or the 'maestro' (Davidson, Yamazaki, and Menaker 2003).

Furthermore, the communication between the conductor and the orchestra is bidirectional. Similarly, the cellular clock has been shown to be in itself regulated by its downstream output processes: e.g., the findings of clock-regulated metabolic cycles feeding back to the circadian system (Asher and Sassone-Corsi 2015; Asher and Schibler 2011).

๑ OPEN ACCESS - PTPBIO.ORG 
In sum, the 'conductor' can voice or deliver entailments which express certain aspects of the system for which the 'clock' does not suffice. As this metaphor is still emerging, other 'conductor' entailments might become more relevant in future research. The conductor's work is not limited to setting the tempo of the piece, and their physical gestures are used as well to control the musical dynamics, accent, and articulation throughout a concert (i.e., " $A$ conductor conveys multiple, parallel messages"). This can be likened to the influence of more state variables of the oscillator, like amplitude and overall pattern, rather than only that of its phase. It also highlights the concept of flexibility: the conductor interprets a score (i.e., "A conductor has artistic freedom"), which can be analogous to the fact that this system can sense and respond to the ever changing biological needs of a cell or a body.

\section{Final Notes}

The limits of my language are the limits of my world.

-Ludwig Wittgenstein (1922)

In this work we traced the history of chronobiology through the perspective of the metaphorical language used in scientific literature. We found that chronobiology research was influenced by the use of metaphors, predominantly by that of the 'clock', since it was adopted throughout the 1950s. The 'clock' metaphor served as a significant unifying theme, alongside the establishment of the chronobiology field.

We further examined the usefulness that the 'clock' metaphor served by describing its entailments, namely associative implications of the metaphor. These entailments served two main purposes: (1) providing an explanatory mean: introducing new meaning to the experimental observations, thereby enabling explanation of an elusive phenomenon in terms of a well-known one; and (2) providing a predictive mean: by explicitly inspiring experiments which became milestones in the development of chronobiology field.

Analysis of the different metaphors reveals an additional role: (3) metaphors and entailments can serve as defining means and shape the field's paradigm. The choice of a certain metaphor can determine what are the important questions, what are the tools needed to answer these questions, what would be the legitimate range of answers, and can also hide (i.e., divert the scientific endeavor or attention from) the aspects of reality that are not included in the above.

Here we used metaphors to revisit past discovery paths, while contemporary analyses are more challenging and rely on speculations. We take the liberty of offering one such venture: Today, the clock field is heavily leaning towards translational research (i.e., studies that bear a direct/potential benefit for medicine or well-being), (Panda 2019; Manoogian, Chaix, and Panda 2019; Ruben, Hogenesch, and Smith 2019; Z. Liu and Chu 2013). Although this trend is not unique to the clock field, it does go hand in hand with the 'conductor' metaphor: saying that the clock is a 'conductor' bestows upon it the glory of a valuable leader, suggesting things might go wrong without it. When the system is seen as an orchestra, the coordination and alignment between its parts become much more important. Along these lines, the concept of internal desynchrony as a cause of pathology attracted much attention in recent years (West and Bechtold 2015; Roenneberg and Merrow 2016; Nicholls et al. 2019; Manella et al. 2020), although, curiously, so far there has been no direct evidence to support this idea (see van der Vinne et al. 2018).

Our research shows that over the years different metaphors were suggested prior to and in parallel with the 'clock'. Interestingly, these share a defining characteristic in that they are "telosgivers": each metaphor entails a certain purpose for the system, and this purpose is essential

๑ OPEN ACCESS - PTPBIO.ORG 
for the metaphor's implementation. Be it time-measurement, memory, or coordination, this purpose has helped scientists construct their view of the system. Telos-giving metaphors appear in other domains of biology as well. For example, information-relay is the purpose entailed by metaphors like 'signaling' or 'translation'. Regarding molecules as 'messengers' is not inherent in their chemical/materialistic properties, but rather stems from their "role" as understood by scientists. Therefore, despite the exclusion of teleology brought on by the rise of the mechanistic worldview during the Scientific Revolution, themes of guidance and reason are inherent to biological language (see also Riskin 2016). It is our impression that such telos-giving metaphors are widespread in biology, while in other fields such as chemistry and physics they are much less abundant. This could point to an interesting linguistic disciplinary divide, and so calls for further investigation.

Metaphors We Live By (Lakoff and Johnson 1980), which was fundamental for this research, leaves the strong impression that conceptual metaphors are so embedded in people's thought processes that they are normally completely oblivious to them. From this perspective, it is easy to understand the reluctance some authors express regarding the use of metaphors in science as they can lead to misunderstanding and misconceptions (Kampourakis 2016; Taylor and Dewsbury 2018). However, our results point towards a different view of the subject, as not only are metaphors inherent to the scientific discourse and were scientifically fruitful, but also they have often been used consciously and even deliberately by scientists. It appears that those who promoted the use of specific metaphors-e.g., Pittendrigh and the 'clock', Menaker and the 'conductor'-had a clear line of entailment drawing, followed by hypothesis-making and experimentation, even if they didn't necessarily use the word "metaphor" (some alternatives were 'paradigm,' 'model,' 'analogy,' etc.). Pittendrigh even went so far as to expose the weakness of his metaphor and to suggest alternatives.

Of note, such "conscious use" is the most straightforward to identify, because the aware "users" would leave testimonies for their use in writing (while the unaware would not and could not discuss it at all). Such "sample bias" means that, on our part, the effect of "unconscious use" and "transparent" metaphors is less accessible for reflection and understanding. This raises many important questions: How much do this study's conclusions apply to other metaphors in science, or in other words, Is the 'clock' a representative case-study? Can we classify metaphors in science according to the ways they are used? Are there additional metaphors still hidden in plain sight?

\section{Acknowledgments}

We thank Gad Asher, Menachem Fisch, Snait Gissis, Noah Stemeroff, William Swartz, and Yael Gaulan for their guidance, discussions, and perspective. We also thank Erik Bitmann, Ute Deichmann, Anna Wirz-Justice, Elizabeth Klerman, and Shimon Ullman for their valuable comments on the manuscript. Notably, this work could not have been accomplished without the meticulous narration of the field's history by Colin S. Pittendrigh and Serge Daan. We thank Charlie's Cafe and its devoted staff for the longitudinal hospitality and caffeine supply throughout this work. Finally, we are wholeheartedly grateful for Omer Benjakob, whose intellect, insight, and ideas were indispensable for the entire project.

\section{Literature cited}

Anafi, Ron C., Yool Lee, Trey K. Sato, Anand Venkataraman, Chidambaram Ramanathan, Ibrahim H. Kavakli, Michael E. Hughes, et al. 2014. "Machine Learning Helps Iden- 
tify CHRONO as a Circadian Clock Component.” PLoS Biology 12 (4): e1001840. doi:10.1371/journal.pbio.1001840.

Aschoff, J. 1960. "Exogenous and Endogenous Components in Circadian Rhythms." Cold Spring Harbor Symposia on Quantitative Biology 25 (0): 11-28. doi:10.1101/SQB.1960.025.01.004.

Aschoff, J., and R. Wever. 1976. "Human Circadian Rhythms: A Multioscillatory System." Federation Proceedings 35 (12): 236-232.

Asher, Gad, and Paolo Sassone-Corsi. 2015. "Time for Food: The Intimate Interplay between Nutrition, Metabolism, and the Circadian Clock.” Cell 161 (1): 84-92. doi:10.1016/j.cell.2015.03.015.

Asher, Gad, and Ueli Schibler. 2011. "Crosstalk between Components of Circadian and Metabolic Cycles in Mammals.” Cell Metabolism 13 (2): 125-37. doi:10.1016/j.cmet.2011.01.006.

Balsalobre, A., F. Damiola, and U. Schibler. 1998. "A Serum Shock Induces Circadian Gene Expression in Mammalian Tissue Culture Cells." Cell 93 (6): 929-37.

Beling, Ingeborg. 1929. "Über das Zeitgedächtnis der Bienen.” Zeitschrift für vergleichende Physiologie 9 (2): 259-338. doi:10.1007/BF00340159.

Black, Max. 1962. Models and Metaphors: Studies in Language and Philosophy. 7th printing. Ithaca: Cornell Univ. Press.

—. 1979. "More about Metaphor." In Metaphor and Thought, edited by Andrew Ortony. Cambridge University Press, Cambridge, England.

Boyd, Richard. 1979. “Metaphor and Theory Change: What Is 'Metaphor' a Metaphor For?” In Metaphor and Thought, edited by Andrew Ortony, 481-532.

Brown, Theodore L. 2003. Making Truth: Metaphor in Science. Urbana: University of Illinois Press.

Buhr, Ethan D., and Joseph S. Takahashi. 2013. "Molecular Components of the Mammalian Circadian Clock." Handbook of Experimental Pharmacology 217: 3-27. doi:10.1007/978-3-642-25950$0 \_1$.

Bunning, E. 1960. “Opening Address: Biological Clocks.” Cold Spring Harbor Symposia on Quantitative Biology 25 (0): 1-9. doi:10.1101/SQB.1960.025.01.003.

Carriero, John, Paul Hoffman, John Cottingham, Robert Stoothoff, and Dugald Murdoch. 1990. "The Philosophical Writings of Descartes.” The Philosophical Review 99 (1): 93. doi:10.2307/2185205.

Cold Spring Harbor Laboratory of Quantitative Biology. 1960. Biological Clocks. Vol. 25. Biological Laboratory.

Daan, Serge. 2010. "A History of Chronobiological Concepts." In The Circadian Clock, edited by Urs Albrecht, 1-35. New York, NY: Springer. doi:10.1007/978-1-4419-1262-6_1.

Davidson, Alec J., Shin Yamazaki, and Michael Menaker. 2003. "SCN: Ringmaster of the Circadian Circus or Conductor of the Circadian Orchestra?" Novartis Foundation Symposium 253: 110-21; discussion 121-125, 281-84.

De Mairan, J.-J. 1729. “Observation Botanique.” Histoire de l'Academie Royale Sciences 35: 36.

Dibner, Charna, Ueli Schibler, and Urs Albrecht. 2010. “The Mammalian Circadian Timing System: Organization and Coordination of Central and Peripheral Clocks." Annual Review of Physiology 72 (1): 517-49. doi:10.1146/annurev-physiol-021909-135821.

Dolnick, Edward. 2014. Isaac Newton, the Royal Society, and the Birth of the Modern World. HarperCollins e-Books. http://rbdigital.oneclickdigital.com.

Enright, J. T. 1975. "The Circadian Tape Recorder and Its Entrainment." In Physiological Adaptation to the Environment, edited by F. J. Vernberg, 465-76. New York: Intext Educational Publishers. 
Haber, F. C. 1975. "The Cathedral Clock and the Cosmological Clock Metaphor." In The Study of Time II: Proceedings of the Second Conference of the International Society for the Study of Time Lake Yamanaka-Japan, edited by J. T. Fraser and N. Lawrence, 399-416. Berlin, Heidelberg: Springer Berlin Heidelberg. doi:10.1007/978-3-642-50121-0_30.

Hardin, P. E., J. C. Hall, and M. Rosbash. 1990. "Feedback of the Drosophila Period Gene Product on Circadian Cycling of Its Messenger RNA Levels.” Nature 343 (6258): 536-40. doi:10.1038/343536a0.

Hastings, J. W., and B. M. Sweeney. 1957. "On the Mechanism of Temperature Independence in a Biological Clock." Proceedings of the National Academy of Sciences of the United States of America 43 (9): 804-11. doi:10.1073/pnas.43.9.804.

Hastings, J. Woodland. 2001. "Fifty Years of Fun.” Journal of Biological Rhythms 16 (1): 5-18. doi:10.1177/074873040101600102.

Hesse, Mary B. 1970. Models and Analogies in Science. Notre Dame, Ind.: University of Notre Dame Press.

Johnson, Carl Hirschie. 1992. "Phase Response Curves: What Can They Tell Us about Circadian Clocks.” In Circadian Clocks from Cell to Human, edited by T. Hiroshige and K. Honma, 209-49.

Johnson, Maynard S. 1939. "Effect of Continuous Light on Periodic Spontaneous Activity of White-Footed Mice (Peromyscus)." Journal of Experimental Zoology 82 (2): 315-28. doi:10.1002/jez.1400820209.

Kalmus, H., and L. A. Wigglesworth. 1960. "Shock Excited Systems as Models for Biological Rhythms.” Cold Spring Harbor Symposia on Quantitative Biology 25 (0): 211-16. doi:10.1101/SQB.1960.025.01.020.

Kalmus, Hans. 1940. "New Research in the Diurnal Periodicity of Animals." Acta Medica Scandinavica 103 (S108): 227-33. doi:10.1111/j.0954-6820.1940.tb11096.x.

- 1988. "The History and Philosophy of Chronobiology." Journal of Interdisiplinary Cycle Research 19 (4): 227-34. doi:10.1080/09291018809359981.

Kampourakis, Kostas. 2016. “The Bad Use of Metaphors and the Use of Bad Metaphors.” Science \& Education 25 (9-10): 947-49. doi:10.1007/s11191-016-9870-2.

Kramer, Gustav. 1952. “Experiments on Bird Orientation.” Ibis 94 (2): 265-85. doi:10.1111/j.1474919X.1952.tb01817.x.

Lakoff, George, and Mark Johnson. 1980. Metaphors We Live By. Chicago, Ill.: Univ. of Chicago Press.

Liu, Y., N. F. Tsinoremas, C. H. Johnson, N. V. Lebedeva, S. S. Golden, M. Ishiura, and T. Kondo. 1995. "Circadian Orchestration of Gene Expression in Cyanobacteria." Genes E Development 9 (12): 1469-78. doi:10.1101/gad.9.12.1469.

Liu, Zhihua, and Guiyan Chu. 2013. "Chronobiology in Mammalian Health.” Molecular Biology Reports 40 (3): 2491-2501. doi:10.1007/s11033-012-2330-4.

Manella, Gal, Rona Aviram, Nityanand Bolshette, Sapir Muvkadi, Marina Golik, David F. Smith, and Gad Asher. 2020. "Hypoxia Induces a Time- and Tissue-Specific Response That Elicits Intertissue Circadian Clock Misalignment." Proceedings of the National Academy of Sciences 117 (1): 779-86. doi:10.1073/pnas.1914112117.

Manoogian, Emily N. C., Amandine Chaix, and Satchidananda Panda. 2019. "When to Eat: The Importance of Eating Patterns in Health and Disease.” Journal of Biological Rhythms 34 (6): 57981. doi:10.1177/0748730419892105. 
Mauvoisin, Daniel, Florian Atger, Loïc Dayon, Antonio Núñez Galindo, Jingkui Wang, Eva Martin, Laetitia Da Silva, et al. 2017. "Circadian and Feeding Rhythms Orchestrate the Diurnal Liver Acetylome.” Cell Reports 20 (7): 1729-43. doi:10.1016/j.celrep.2017.07.065.

Mayr, Ernst. 1961. "Cause and Effect in Biology.” Science 134 (3489): 1501-6. http://www.jstor.org/stable/1707986.

Moore, Robert Y., and Victor B. Eichler. 1972. "Loss of a Circadian Adrenal Corticosterone Rhythm Following Suprachiasmatic Lesions in the Rat.” Brain Research 42 (1): 201-6. doi:10.1016/00068993(72)90054-6.

Morse, David, J. Woodland Hastings, and Till Roenneberg. 1994. "Different Phase Responses of the Two Circadian Oscillators in Gonyaulax." Journal of Biological Rhythms 9 (3-4): 263-74. doi: $10.1177 / 074873049400900307$.

Nagoshi, Emi, Camille Saini, Christoph Bauer, Thierry Laroche, Felix Naef, and Ueli Schibler. 2004. "Circadian Gene Expression in Individual Fibroblasts: Cell-Autonomous and Self-Sustained Oscillators Pass Time to Daughter Cells." Cell 119 (5): 693-705. doi:10.1016/j.cell.2004.11.015.

Nicholls, Shannon K., Leandro P. Casiraghi, Wanqi Wang, E. Todd Weber, and Mary E. Harrington. 2019. "Evidence for Internal Desynchrony Caused by Circadian Clock Resetting." The Yale Journal of Biology and Medicine 92 (2): 259-70.

Panda, Satchidananda. 2019. “The Arrival of Circadian Medicine.” Nature Reviews Endocrinology 15 (2): 67-69. doi:10.1038/s41574-018-0142-x.

Pett, J. Patrick, Anja Korenčič, Felix Wesener, Achim Kramer, and Hanspeter Herzel. 2016. "Feedback Loops of the Mammalian Circadian Clock Constitute Repressilator.” PLOS Computational Biology 12 (12): e1005266. doi:10.1371/journal.pcbi.1005266.

Pittendrigh, C. S. 1954. "On Temperature Independence in the Clock System Controlling Emergence Time in Drosophila." Proceedings of the National Academy of Sciences of the United States of America 40 (10): 1018-29. doi:10.1073/pnas.40.10.1018.

- 1960. "Circadian Rhythms and the Circadian Organization of Living Systems." Cold Spring Harbor Symposia on Quantitative Biology 25: 159-84.

- 1993. "Temporal Organization: Reflections of a Darwinian Clock-Watcher." Annual Review of Physiology 55: 16-54. doi:10.1146/annurev.ph.55.030193.000313.

- 1996. "'Lecture' to Friends."

Reddy, Akhilesh B., Natasha A. Karp, Elizabeth S. Maywood, Elizabeth A. Sage, Michael Deery, John S. O’Neill, Gabriel K. Y. Wong, et al. 2006. "Circadian Orchestration of the Hepatic Proteome.” Current Biology 16 (11): 1107-15. doi:10.1016/j.cub.2006.04.026.

Reddy, P., W. A. Zehring, D. A. Wheeler, V. Pirrotta, C. Hadfield, J. C. Hall, and M. Rosbash. 1984. "Molecular Analysis of the Period Locus in Drosophila melanogaster and Identification of a Transcript Involved in Biological Rhythms.” Cell 38 (3): 701-10.

Reinberg, Alain E., Hadas Lewy, and Michael Smolensky. 2001. "The Birth of Chronobiology: Julien Joseph Virey 1814.” Chronobiology International 18 (2): 173-86. doi:10.1081/CBI-100103184.

Renner, M. 1960. "The Contribution of the Honey Bee to the Study of Time-Sense and Astronomical Orientation." Cold Spring Harbor Symposia on Quantitative Biology 25: 361-67.

Riskin, Jessica. 2016. The Restless Clock: A History of the Centuries-Long Argument over What Makes Living Things Tick. Chicago: The University of Chicago Press.

Roenneberg, Till, and Martha Merrow. 2016. “The Circadian Clock and Human Health.” Current Biology 26 (10): R432-43. doi:10.1016/j.cub.2016.04.011.

๑ OPEN ACCESS - PTPBIO.ORG 
Rosbash, M., and J. C. Hall. 1989. “The Molecular Biology of Circadian Rhythms.” Neuron 3 (4): 387-98.

Ruben, Marc D., John B. Hogenesch, and David F. Smith. 2019. "Sleep and Circadian Medicine.” Neurologic Clinics 37 (3): 615-29. doi:10.1016/j.ncl.2019.03.004.

Schwartz, William J., and Serge Daan. 2017. “Origins: A Brief Account of the Ancestry of Circadian Biology.” In Biological Timekeeping: Clocks, Rhythms and Behaviour, edited by Vinod Kumar, 3-22. New Delhi: Springer India. doi:10.1007/978-81-322-3688-7_1.

Sobel, Dava. 2007. Longitude: The True Story of a Lone Genius Who Solved the Greatest Scientific Problem of His Time. New York: Walker.

Stephan, F. K., and I. Zucker. 1972. "Circadian Rhythms in Drinking Behavior and Locomotor Activity of Rats Are Eliminated by Hypothalamic Lesions." Proceedings of the National Academy of Sciences of the United States of America 69 (6): 1583-86. doi:10.1073/pnas.69.6.1583.

Takahashi, Joseph S. 2004. "Finding New Clock Components: Past and Future." Journal of Biological Rhythms 19 (5): 339-47. doi:10.1177/0748730404269151.

Takahashi, Joseph S., and Michael Menaker. 1982. "Entrainment of the Circadian System of the House Sparrow: A Population of Oscillators in Pinealectomized Birds." Journal of Comparative Physiology 146 (2): 245-53. doi:10.1007/BF00610244.

Takahashi, Joseph S., Fred W. Turek, and Robert Y. Moore, eds. 2001. Handbook of Behavioral Neurobiology. Vol. 12. Boston, MA: Springer US. doi:10.1007/978-1-4615-1201-1.

Taylor, Cynthia, and Bryan M. Dewsbury. 2018. "On the Problem and Promise of Metaphor Use in Science and Science Communication.” Journal of Microbiology \& Biology Education 19 (1). doi:10.1128/jmbe.v19i1.1538.

Thurley, Kevin, Christopher Herbst, Felix Wesener, Barbara Koller, Thomas Wallach, Bert Maier, Achim Kramer, and Pål O. Westermark. 2017. "Principles for Circadian Orchestration of Metabolic Pathways." Proceedings of the National Academy of Sciences 114 (7): 1572-77. doi:10.1073/pnas.1613103114.

Vinne, Vincent van der, Steven J. Swoap, Thomas J. Vajtay, and David R. Weaver. 2018. "Desynchrony between Brain and Peripheral Clocks Caused by CK1 $/ \varepsilon$ Disruption in GABA Neurons Does Not Lead to Adverse Metabolic Outcomes." Proceedings of the National Academy of Sciences 115 (10): E2437-46. doi:10.1073/pnas.1712324115.

Virey, Julien-Joseph. 1814. Ephémérides de la vie humaine, ou recherches sur la revolution journalière et la periodicité des ses phenomènes dans la santé et les maladies; thèse, etc. Paris: Didot Jeune.

West, Alexander C., and David A. Bechtold. 2015. "The Cost of Circadian Desynchrony: Evidence, Insights and Open Questions.” BioEssays 37 (7): 777-88. doi:10.1002/bies.201400173.

Westfall, R. S., G. Basalla, and O. Hannaway. 1977. The Construction of Modern Science: Mechanisms and Mechanics. Cambridge University Press. https://books.google.co.il/books?id= ED76ljJ6CD0C.

Winfree, A. T. 2001. The Geometry of Biological Time. New York: Springer. https://books.google.co.il// books?id=5YktgBuoglAC.

Yoo, Seung-Hee, Shin Yamazaki, Phillip L. Lowrey, Kazuhiro Shimomura, Caroline H. Ko, Ethan D. Buhr, Sandra M. Siepka, et al. 2004. "PERIOD2::LUCIFERASE Real-Time Reporting of Circadian Dynamics Reveals Persistent Circadian Oscillations in Mouse Peripheral Tissues." Proceedings of the National Academy of Sciences of the United States of America 101 (15): 5339-46. doi:10.1073/pnas.0308709101. 
(C) 2020 Author(s)

This is an open-access article distributed under the terms of the Creative Commons Attribution 4.0 International license, which permits anyone to download, copy, distribute, display, or adapt the text without asking for permission, provided that the creator(s) are given full credit.

ISSN 2475-3025 